\title{
UTILIZAÇÃO DO PROGRAMA COMPUTACIONAL COMSOL MULTIPHYSICS PARA OBTENÇÃO DE PERFIL DE VELOCIDADE EM REGIME DE FLUXO COM DESLIZAMENTO
}

\author{
G. S. MARQUES, M. DeSOUZA e S. H. de FARIA \\ Universidade Estadual de Maringá, Departamento de Engenharia Química \\ e-mail para contato: geovana.smarques@gmail.com
}

\begin{abstract}
RESUMO - O escoamento em microcanais difere do escoamento em sistemas macroscópicos. A aplicabilidade das equações de Navier-Stokes em microescala é discutível, pois fenômenos como a tensão superficial e a rugosidade da superfície quando comparados às dimensões do canal assumem papéis importantes. Um microcanal é caracterizado por suas dimensões micrométricas e o escoamento em seu interior geralmente ocorre no regime de escoamento com deslizamento, no qual a velocidade do fluido na parede é diferente da velocidade da parede, apesar do fluido se manter como um continuum no seio do fluxo. Nesse regime, a equação de NavierStokes pode ser usada, mas com condições de contorno modificadas. Este trabalho teve como objetivo utilizar o programa comercial COMSOL ${ }^{T M}$ Multiphysics para descrever os perfis de velocidade de um fluido escoando no regime de deslizamento entre duas placas paralelas usando a condição de deslizamento de Maxwell. Os resultados obtidos foram comparados com aqueles apresentados pela equação de Beskok e Karniadakis a fim de se verificar sua validade, e mostraram-se satisfatórios.
\end{abstract}

\section{INTRODUÇÃO}

Microrreatores ou reatores de microcanais são equipamentos que possuem canais de escoamento com dimensões da ordem de dezenas a centenas de mícrons de largura por alguns centímetros de comprimento e caracterizam-se principalmente por sua elevada área superficial específica, que lhes confere altas taxas de transferência de massa e de energia. A atual tecnologia de microfabricação permite a construção de sistemas micrométricos de elevada complexidade com várias operações unitárias integradas (Zhou et al., 2014). Tem-se observado que o comportamento do escoamento de fluidos nesses microcanais possui características bastante peculiares, quando comparado aos sistemas macroscópicos (Arkilic et al., 1997). Os modelos matemáticos que descrevem esse comportamento variam em complexidade, e a validade das tradicionais equações de Navier-Stokes não é assegurada. Vários fenômenos, tais como a tensão superficial e a relação entre a magnitude relativa da rugosidade da superfície e as dimensões do canal, que não são muito significativos em macroescala, contribuem para tal observação. 
O escoamento de gás rarefeito em canais, canos e dutos com superfícies lisas têm sido estudado em uma ampla faixa do número de Knudsen $(K n)$ a baixo número de Mach $(M a)$ com o propósito de desenvolver modelos simples com base nos fenômenos físicos. Tais fluxos são encontrados em ambientes de baixa pressão e em ambientes com geometria em escalas micrométricas ou inferiores, em condições atmosféricas padrão. Os números de Knudsen e de Mach são definidos pelas Equações 1 e 2, respectivamente.

$$
\begin{gathered}
K n=\frac{\lambda}{H} \\
M a=\frac{v}{c}
\end{gathered}
$$

Onde $\lambda$ é o livre percurso médio das moléculas do gás, $H$ a dimensão característica do canal, $v$ a velocidade do fluido, e $c$ a velocidade do som.

A primeira categoria inclui aplicações em equipamentos usados em vôos supersônicos e vários tipos de instrumentos a vácuo. Aplicações na segunda categoria são encontradas em MEMS, equipamento em microescala que associa processos eletrônicos e mecânicos em um sistema único, e inclui instrumentação, equipamentos microeletrônicos, bioengenharia e microrreatores, onde complexas redes de microcanais são usadas e a velocidade característica situa-se na faixa de $0,01 \mathrm{~m} / \mathrm{s}$ a $1,00 \mathrm{~m} / \mathrm{s}$, muito menor que a velocidade do som, que em temperatura ambiente é de aproximadamente $340 \mathrm{~m} / \mathrm{s}$. Nessas aplicações, em geral, o fluxo de gás não pode ser modelado com base na teoria do continuum. O livre percurso médio das moléculas, que na atmosfera padrão é de aproximadamente $65 \mathrm{~nm}$, é comparável à dimensão característica do canal, e por isso, os efeitos microscópicos são importantes. O desvio do estado continuum do gás é medido pelo número de Knudsen $(K n)$. Dutos com largura de $100 \mathrm{~nm}$ ou menos, que atualmente são comuns nas aplicações da nanotecnologia, correspondem a $K n \approx 1$. Em outros fluxos capilares, tais como em equipamentos de detecção da fuga de Hélio e espectrofotômetro de massa, Kn pode atingir valores próximos a 200 (Arkilic et al., 1997; Beskok e Karniadakis, 1999; Choi et al., 2003).

À medida que o número de Knudsen aumenta, os efeitos da rarefação tornam-se mais importantes e a queda de pressão, a tensão de cisalhamento, o fluxo de calor e a taxa de fluxo mássico não podem mais ser preditos pelos modelos de transporte tradicionais baseados na hipótese do continuum. Por outro lado, modelos simples baseados nos conceitos da teoria cinética dos gases também não são apropriados, exceto no caso de $K n \gg 1$, correspondente a condições muito próximas do vácuo total. Os modelos de fluxo apropriados dependem da faixa do número de Knudsen: para $K n \leq 10^{-3}$ o fluido pode ser considerado um continuum, para valores de $K n$ entre $10^{-3}$ e $10^{-1}$ o fluxo é classificado como escoamento com deslizamento, para $10^{-1} \leq \mathrm{Kn}<10$ o fluxo é chamado de escoamento de transição, enquanto para $K n \geq 10$ o fluxo é denominado fluxo molecular livre (Beskok e Karniadakis, 1999; Pinazza e Spiga, 2003).

Trabalhos experimentais e teóricos demonstram que no escoamento com deslizamento existe uma dependência não linear entre a taxa de fluxo mássico e a queda de pressão. Essa dependência 
é mais bem relacionada com o quadrado da diferença entre as pressões de entrada e saída, que é característica de fluxos compressíveis em longos dutos.

No escoamento com deslizamento $\left(10^{-3}<K n<10^{-1}\right)$, a velocidade do fluido na parede é diferente da velocidade da parede, apesar do fluido se manter como um continuum no seio do fluxo. Nesse regime, a equação de Navier-Stokes pode ser usada, mas com condições de contorno modificadas. Vários trabalhos que tratam dessas condições de contorno modificadas estão sendo propostas, ou com base nos argumentos da teoria cinética, ou a partir de uma análise da equação de Boltzmann; mas, em ambos os casos, severas simplificações relacionadas à dinâmica do sistema são necessárias. A condição de contorno modificada mais simples é aquela que introduz uma dependência linear entre a velocidade de deslizamento na parede do canal (deslizamento parcial) e o gradiente de velocidade, conhecida também como condição de contorno de deslizamento de primeira ordem em relação ao número de Knudsen (Pinazza e Spiga, 2003).

A condição de deslizamento de Maxwell tem sido amplamente utilizada para predizer a velocidade de deslizamento na parede. Essa condição é baseada na interação molecular entre o gás e a superfície do canal descrita pela teoria cinética dos gases. O grau de não elasticidade da colisão entre as moléculas do gás e a parede é descrita pelo coeficiente tangencial de acomodação. Supondo fluxo isotérmico, uma correção de primeira ordem no número de Knudsen pode ser escrita como (Arkilic et al., 1997):

$$
u_{g p}-u_{w}=\frac{2-\sigma_{\mathrm{v}}}{\sigma_{\mathrm{v}}}\left[\left.K n \frac{\partial u}{\partial y}\right|_{w}\right]
$$

Em que $u_{g p}$ é a velocidade do gás junto à superfície do canal; $u_{w}$ é a velocidade da superfície do canal, geralmente nula; $\sigma_{v}$ é o coeficiente de acomodação tangencial; $\left.\frac{\partial u}{\partial y}\right|_{w}$ é o gradiente da velocidade normal à superfície do canal; e $y$, a direção normal ao fluxo, normalizada pela dimensão característica $H$, isto é, $y=Y / H$.

Como o coeficiente $\sigma_{\mathrm{v}}$ não considera adequadamente a diferença entre um gás monoatômico e outros, Aubert e Colin (2001) e Beskok e Karniadakis (1999) desenvolveram modelos para a condição de contorno de deslizamento com correção de segunda-ordem para o número de Knudsen; entretanto, esses modelos incluem a desvantagem da dificuldade de se calcular o termo gradiente de segunda ordem. Caso o termo dentro dos colchetes na Equação 3 seja considerado como uma expansão em série de Taylor truncada no primeiro termo, uma condição de contorno de deslizamento de ordem mais elevada poderá ser obtida pela seguinte equação (Beskok e Karniadakis, 1999):

$$
u_{g p}-u_{w}=\frac{2-\sigma_{\mathrm{v}}}{\sigma_{\mathrm{v}}}\left[\left.K n \frac{\partial u}{\partial y}\right|_{w}+\left.\frac{K n^{2}}{2} \frac{\partial^{2} u}{\partial y^{2}}\right|_{w}+\ldots\right]
$$


Para suprir os problemas do modelo de Maxwell e do modelo de Beskok e Karniadakis (1999), outros trabalhos foram desenvolvidos utilizando o modelo de Langmuir, que tem origem nas teorias da química de superfícies, e no modelo de Leonard-Jones, com suposições de paredes atrativas ou repulsivas (Choi et al., 2003).

Beskok e Karniadakis (1999) propuseram um modelo de fluxo laminar entre placas paralelas para o escoamento com deslizamento, que inclui uma correção de segunda-ordem para o número de Knudsen. Esse perfil foi validado por meio do Simulador de Monte Carlo (um método estatístico para simulações estocásticas com diversas aplicações em áreas como a física, a matemática e a biologia) utilizando-se de dados experimentais, e é dado por:

$$
\frac{U(y)}{\bar{U}}=\frac{-(y)^{2}+(y)+\psi}{\frac{1}{6}+\psi}
$$

sendo:

$$
\psi=\left(\frac{2-\sigma_{v}}{\sigma_{v}}\right) \frac{K n}{1-b K n}
$$

e $b$, um parâmetro empírico denominado de coeficiente de deslizamento; $U$ a velocidade do fluido na direção do eixo $x ; \bar{U}=\bar{U}(X)$ a velocidade média local do fluido na direção $x$; e $y$ a coordenada transversal ao fluxo (adimensional).

O presente trabalho consistiu em utilizar o programa comercial COMSOL $^{T M}$ Multiphysics para descrever os perfis de velocidade de um fluido escoando no regime de deslizamento entre duas placas paralelas utilizando a condição de deslizamento de Maxwell. Os resultados obtidos foram comparados com aqueles obtidos pela equação de Beskok e Karniadakis, a fim de se verificar sua validade.

\section{METODOLOGIA}

A fim de se alcançar os objetivos propostos neste trabalho, fez-se necessário realizar uma revisão bibliográfica, buscando-se as leis físicas que governam o fenômeno do escoamento em regime de fluxo com deslizamento, e um estudo do programa COMSOL ${ }^{T M}$ Multiphysics. O segundo passo consistiu em reproduzir geometricamente, na janela apropriada do programa, o processo a ser simulado. Foram reproduzidos, também, processos anteriormente simulados por outros autores. As equações governantes foram, então, transcritas na linguagem requerida pelo programa, e a malha computacional foi definida de maneira a ser suficientemente refinada, para representar adequadamente a continuidade da solução. Feita a construção do modelo matemático para o problema proposto, implementou-se o modelo. $\mathrm{O}$ último passo consistiu em avaliar os resultados obtidos e realizar os ajustes necessários.

Para obtenção do perfil de velocidade de um fluido escoando no regime de deslizamento 
entre duas placas, considerou-se o gás hidrogênio fluindo a uma temperatura de $600 \mathrm{~K}$, com uma velocidade média de $0,03 \mathrm{~m} / \mathrm{s}$, e um microcanal bidimensional de $50 \mu \mathrm{m}$ de altura por $2,5 \mathrm{~cm}$ de comprimento. Os perfis de velocidade foram comparados, também, com aqueles obtidos a partir da condição de contorno de não-deslizamento.

\section{RESULTADOS E DISCUSSÃO}

A fim de se verificar a validade do perfil de velocidade proposto por Beskok e Karniadakis (1999) nos modelos com regime de fluxo com deslizamento, foram comparados os resultados obtidos a partir desse perfil com a solução numérica da equação de Navier-Stokes para a condição de deslizamento de primeira ordem (Equação 3).

Para o gás hidrogênio fluindo a uma temperatura de $600 \mathrm{~K}$, com uma velocidade média de $0,03 \mathrm{~m} / \mathrm{s}$ em um microcanal bidimensional de $50 \mu \mathrm{m}$ de altura por $2,5 \mathrm{~cm}$ de comprimento, o número de Knudsen calculado pela Equação 1 é $\mathrm{Kn} \approx 0,0094$, caracterizando assim, o escoamento com deslizamento. A Figura 1 mostra os perfis de velocidade para os dois casos.

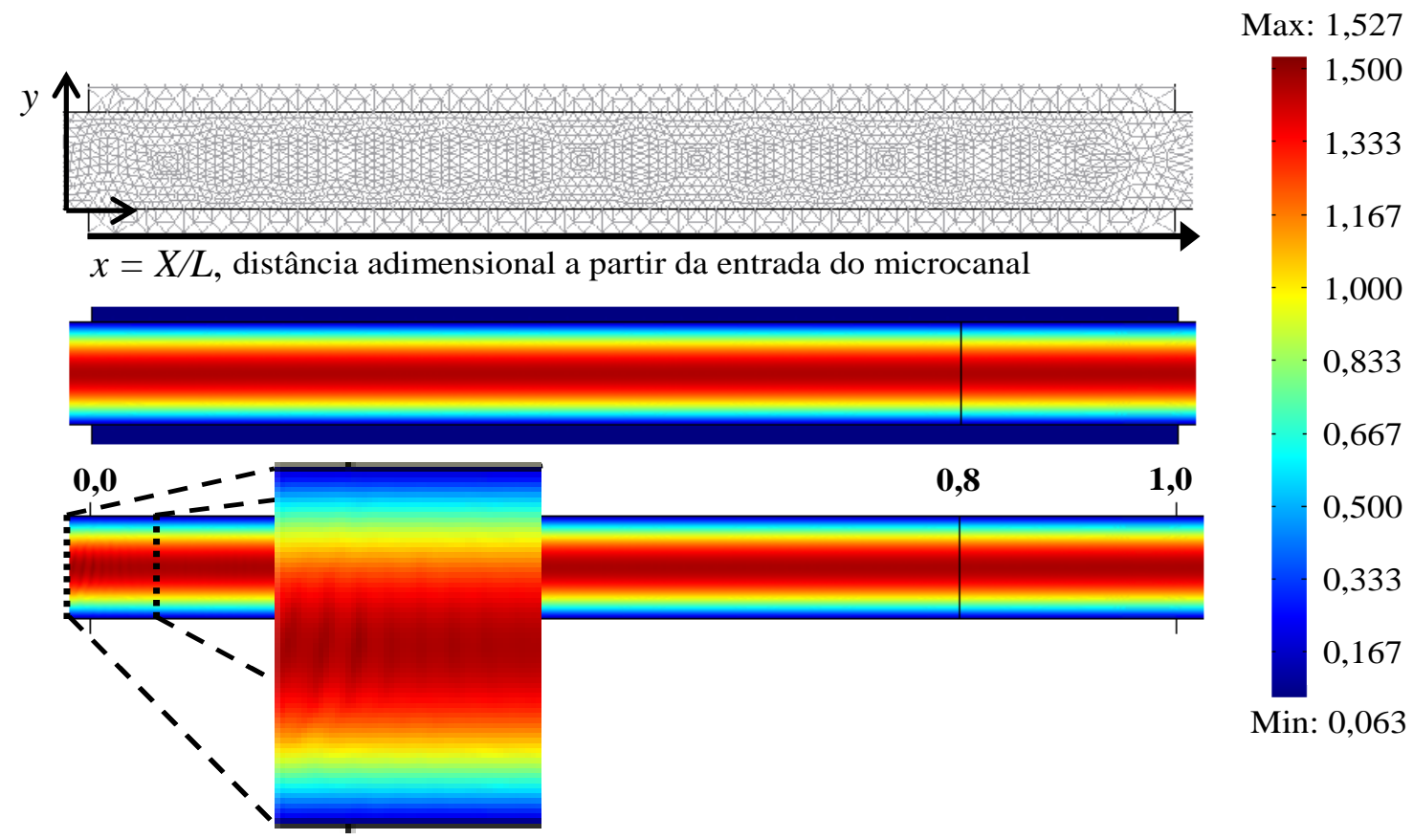

Figura 1 - Velocidade adimensional do $\mathrm{H}_{2}$ no escoamento com deslizamento. $\mathrm{Kn}=0,0094$;

$\mathrm{T}=600 \mathrm{~K}$ e velocidade média de $0,03 \mathrm{~m} / \mathrm{s}$. Comparação entre a solução obtida a partir do COMSOL $^{T M}$ Multiphysics com condição de contorno com deslizamento de primeira ordem e o modelo proposto por Beskok e Karniadakis (1999). (a) Discretização do domínio para o método de elementos finitos: 2261 nós e 4368 elementos. (b) Modelo de Beskok e Karniadakis (1999).

(c) Solução numérica (COMSOL ${ }^{T M}$ Multiphysics). 
É possível observar na solução numérica mostrada na Figura 1-(c), que em uma pequena região localizada na entrada do microcanal ocorre uma oscilação no perfil de velocidade. Essa oscilação deve-se ao fato do perfil ainda não estar totalmente desenvolvido nessa região. A uma distância de 2,0 cm da entrada do canal, onde o perfil de velocidade já está seguramente bem desenvolvido, foi feita a comparação entre os perfis de velocidade propostos por Beskok e Karniadakis e aquele obtido pela solução numérica da equação de Navier-Stokes, cujos resultados são apresentados na Figura 2. Observa-se a partir dessa figura uma boa concordância entre ambos.

O perfil de velocidade para o escoamento do gás hidrogênio com condição de contorno de não-deslizamento nas paredes do microcanal também foi determinado numericamente e os resultados estão apresentados na Figura 2 para fins de comparação com os dois modelos com condição de deslizamento.

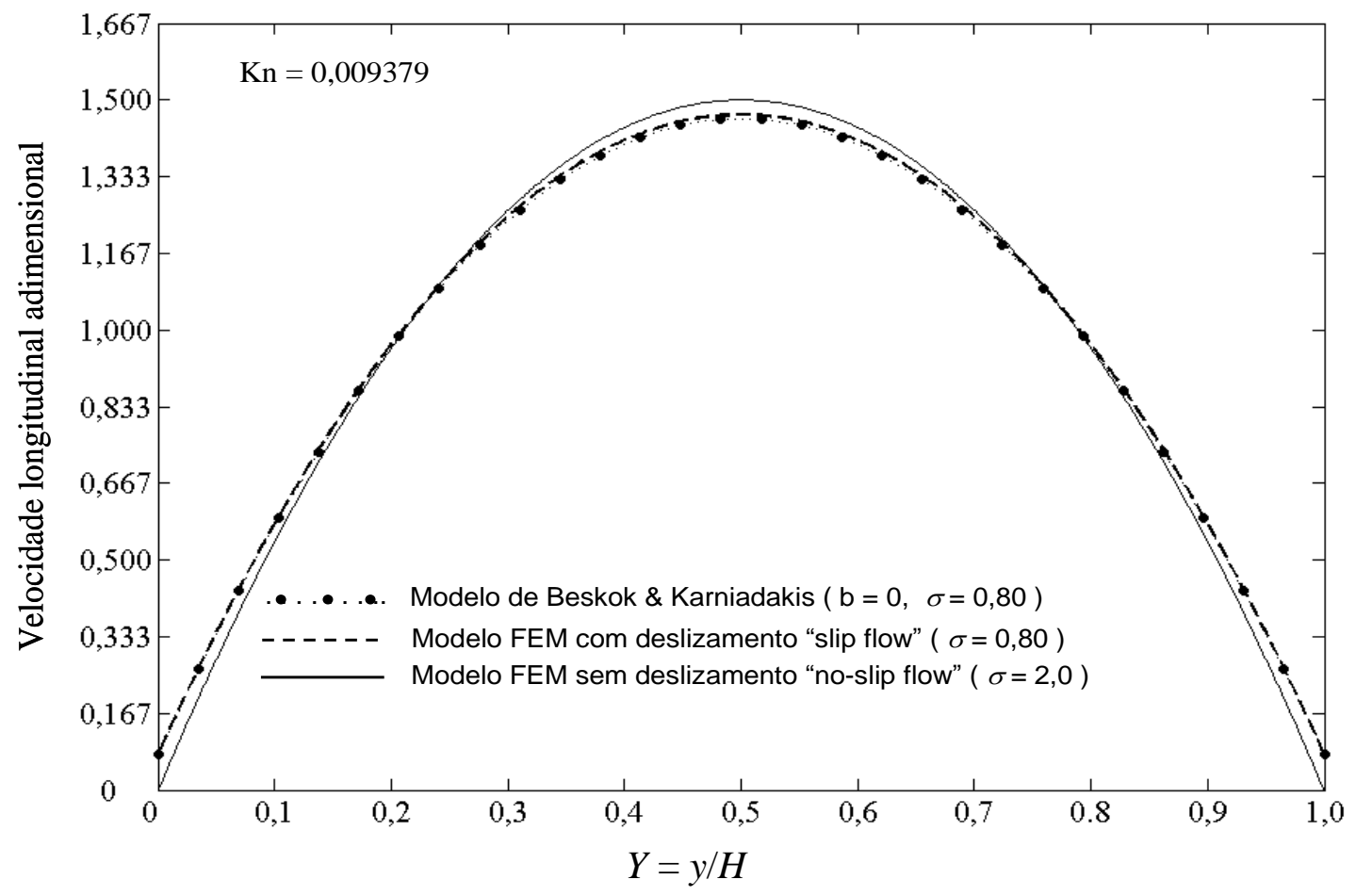

Figura 2 - Perfil de velocidade do fluxo isotérmico com deslizamento a $2,0 \mathrm{~cm}(x=0,8)$ da entrada do microcanal. Comparação entre a solução obtida pelo método numérico do $C O M S O L^{T M}$ Multiphysics e a expressão proposta por Beskok e Karniadakis (1999). T = $600 \mathrm{~K}$, microcanal com $50 \mu \mathrm{m}$ de altura e velocidade média de $0,03 \mathrm{~m} / \mathrm{s}$.

A solução do modelo foi obtida utilizando-se, para o método do programa $C O M S O L^{T M}$ Multiphysics, uma malha com 8430 nós e 14538 elementos. 


\section{CONCLUSÕES}

A partir dos dados obtidos, conclui-se que o perfil de velocidade proposto por Beskok e Karniadakis (1999) apresenta bons resultados, quando comparados àqueles obtidos pela solução numérica da equação de Navier-Stokes. Portanto, é válido e pode ser utilizado para modelagens, implicando, em alguns casos, em uma significativa redução do esforço computacional.

\section{REFERÊNCIAS}

ARKILIC, E.B.; SCHMIDT, M.A.; BREUER, K.S. Gaseous slip flow in long microchannels. J.Microelectromech. S., v.6, p. 167-178, 1997.

AUBERT, C.; COLIN, S. High-order boundary conditions for gaseous flows in rectangular microducts. Microscale Therm. Eng., v. 5, p. 41-54, 2001.

BESKOK, A.; KARNIADAKIS, G.E. A model for flows in channels, pipes, and ducts at micro and nano scales. Microscale Therm. Eng., v. 3, p.43-77, 1999.

CHOI, H.; LEE, D.; MAENG, J. Computation of slip flow in microchannels using Langmuir slip condition. Numer. Heat Tr. A-Appl, v. 44, p. 59-71, 2003.

PINAZZA, O.; SPIGA, M. Friction factor at low Knudsen number for the duct with sine-shaped cross section. Int. J. Heat Fluid Fl., v. 24, p. 236-241, 2003.

ZHOU, W.; DENG, W.; LU, L.; ZHANG, J.; QIN, L.; MA, S.; TANG, Y. Laser micro-milling of microchannel on copper sheet as catalyst support used in microreactor for hydrogen production. Int. J. Hydrogen Energy, v. 39-10, p. 4884-4894, 2014. 